ORIGINAL ARTICLE

\title{
An improved urine collection pad method: a randomised clinical trial
}

\author{
S Rao, J Bhatt, C Houghton, Peter Macfarlane
}

Arch Dis Child 2004;89:773-775. doi: 10.1136/adc.2003.037770

See end of article for authors' affiliations ....................

Correspondence to: Dr Macfarlane, Rotherham General Hospital, Moorgate Road, Rotherham, South Yorkshire S60 2UD, UK; peter.macfarlane@ rothgen.nhs.uk

Accepted 15 October 2003

\begin{abstract}
Aim: To evaluate a modified urine collection pad (UCP) method for its ability to reduce heavy mixed growth bacterial contamination of UCP samples in young children with suspected urinary tract infection (UTI).

Method: Febrile children under 2 years of age were randomised to two UCP methods: the same UCP kept in the nappy until urine was passed (single UCP group), or the UCP replaced with a fresh one every 30 minutes until urine was passed (replaced UCP group). In both groups a moisture sensitive audio alarm was used to signal passage of urine.

Results: Eighty children were enrolled and a satisfactory sample was obtained in 68 (37 in the single UCP group and 31 in the replaced UCP group). In 12 children (15\%), collection failed, mainly because of faecal soiling of the pad. UTI occurred in three children (4\%). In the remaining 65 samples, heavy mixed growth (> $10^{5}$ organisms $/ \mathrm{ml}$ ) occurred in 1/31 (3\%) in the replaced UCP group compared with 10/35 (29\%) in the single UCP group ( $p=0.008$ ). There were no adverse effects from the use of the moisture sensitive audio alarm.

Conclusion: Changing the UCP every 30 minutes almost eliminates heavy mixed growth contamination of UCP samples and substantially increases the proportion of UCP results that confidently exclude UTI. This represents a simple and clinically important improvement to the UCP method which is reliable for diagnosing and excluding UTI in young children still in nappies. It has potential for use in outpatient clinics, in the primary healthcare setting, or at home.
\end{abstract}

rinary tract infection (UTI) is an important cause of fever in young children. Prompt diagnosis and treatment is thought to reduce the risk of renal scarring. ${ }^{1}$ However, the diagnosis is often delayed because of the difficulty in obtaining an uncontaminated urine sample, especially from children who are still in nappies. In the acutely ill child, the preferred sampling method may be suprapubic bladder aspiration but this is invasive and often fails, thereby requiring multiple attempts. Other non-invasive methods, such as clean catch or bag specimens, are difficult, time consuming, and associated with high rates of bacterial contamination by faecal and perineal flora. ${ }^{2}$

The use of urine collection pads (UCPs) is an easy, noninvasive, alternative method of urine sample collection for young children still in nappies in whom UTI needs to be promptly ruled out or diagnosed..$^{3-5}$ Parents prefer this method to both clean catch and bag methods. ${ }^{6}$ However, UCP samples have a high rate of bacterial contamination by skin and faecal flora, limiting their usefulness in ruling out UTI. $^{2}$ In our previous study, ${ }^{7}$ by incorporating a moisture sensitive audio alarm into the UCP, we showed that the heavy mixed growth $\left(>10^{5}\right.$ organisms $\left./ \mathrm{ml}\right)$ contamination of the sample was not related to the length of time the UCP is in contact with the perineal skin after urine has been passed. However, the alarm method was easier to use because the audio alarm signals the immediate presence of urine in the pad eliminating the need to check the nappy regularly.

In this study, we tested the hypothesis that perineal bacterial contamination of the UCP sample could be reduced by minimising the contact time between the dry UCP and perineal skin by comparing frequent replacement (every 30 minutes) with a fresh pad with the standard method of leaving a single pad in situ until urine is passed. We continued to use the moisture sensitive alarm in both groups for convenience and acceptability.

\section{METHOD}

Febrile children under the age of 2 years, admitted to our acute medical wards, in whom the diagnosis of UTI was under consideration, were consecutively invited to take part in the study. After enrolment, the children were randomised to the two urine collection methods: UCP and alarm without replacing the UCP (single UCP group), or UCP and alarm with replacement of the UCP every 30 minutes (replaced UCP group), until urine was passed. The study was performed on two acute general children's wards over an eight month period. Randomisation was achieved by the child's nurse selecting, in sequence, a sealed envelope containing the collection method allocation produced from a computer generated random number list.

We used sterile packed UCPs (Newcastle Urine Collection Pack; Ontex Ltd, Corby, Nottinghamshire, UK; NHS Supplies). The child's perineum was cleaned with soap, rinsed, and dried. The enuresis alarm sensor was cleaned with an alcohol swab before each use.

Wearing gloves, the nurse buried the reusable alarm sensor (Drinite Audio fx 2000 personal enuresis alarm; Ferraris Medical Ltd, Enfield, Middlesex, UK) in the side of the UCP, which in turn was placed inside the front of the child's nappy. The audio alarm was worn attached to the child's clothing or placed at the cot side. In the single UCP group, the same pad was left undisturbed in the nappy until urine was passed. In the replaced UCP group, the pad was replaced every 30 minutes with a new sterile UCP until urine was passed. We used a domestic kitchen timer to alert the child's parent or nurse when each 30 minute time period had elapsed.

When the enuresis alarm signalled passage of urine, the UCP was removed. Pads obviously soiled by faeces were

Abbreviations: UCP, urine collection pads; UTI, urinary tract infection 
discarded and a repeat sample attempted by the same method. Urine was aspirated from the UCP using a $20 \mathrm{ml}$ syringe. The urine sample volume and the time taken to obtain the sample were recorded. A $0.5 \mathrm{ml}$ minimum sample volume was required for bacteriology. The sample was immediately sent for laboratory quantitative bacterial culture, identification, and sensitivity testing (or chilled at $4{ }^{\circ} \mathrm{C}$ until the next day for samples collected at night). Bacteriology laboratory staff analysed and reported the urine bacterial growths blind to the group allocation.

UTI is conventionally defined as a pure growth of a single organism greater than $10^{5} / \mathrm{ml}$. In this study, we considered a mixed growth less than $10^{5} / \mathrm{ml}$ as not indicative of UTI on its own. As the primary outcome measure, we compared the proportions of samples from the two collection methods that were heavily contaminated $\left(>10^{5}\right.$ mixed organisms $/ \mathrm{ml}$ ). This category of results is clinically problematic because UTI cannot definitely be excluded if there is $>10^{5}$ mixed organisms/ml growth. Statistical significance was analysed using a test for comparison of two unpaired proportions.

Our previous study using the standard UCP technique ${ }^{2}$ showed that $27 \%$ of samples were heavily contaminated by skin and faecal flora ( $>10^{5}$ mixed organisms $/ \mathrm{ml}$ ). To show a reduction in the proportion of samples heavily contaminated by half, at significant levels $\mathrm{p}<0.05$, and with $80 \%$ power, we estimated a sample size of 35 children in each group. In our previous studies, ${ }^{27}$ about $20 \%$ of attempts at urine collection failed or were abandoned for various reasons. We therefore aimed to recruit 100 children to the study (50 in each group).

Parents were given an explanatory information sheet about the study, and written consent was obtained. The study protocol was approved by the local research ethics committee.

\section{RESULTS}

Figure 1 summarises the results. Eighty children were recruited and randomised to the two urine collection methods. Urine collection failed in 12 children (15\%, five from the single UCP group and seven from the replaced UCP group) mainly because of faecal soiling of the pad-for example, diarrhoea-leaving 68 children ( 38 boys, 30 girls). A total of 37 samples were collected by the single UCP method and 31 by the replaced UCP method. The age of the children in the two groups was similar, but boys were significantly overrepresented $(\mathrm{p}=0.034)$ in the single UCP group $(25 / 37,68 \%)$, compared with the replaced UCP group $(13 / 31,42 \%)$.

UTI ( $>10^{5}$ organisms/ml pure single organism growth) occurred in 3/68 (4\%) of children. Among the remaining samples, heavy mixed growth $\left(>10^{5}\right.$ mixed growth organisms $/ \mathrm{ml}$ ) was significantly higher in the single UCP group $(10 / 35 ; 29 \%)$ than in the replaced UCP group $(1 / 30 ; 3 \%)$ $(\mathrm{p}=0.008)$. This represents a significant and clinically useful reduction in the proportion of the urine samples yielding a heavy mixed growth result from which UTI cannot be definitely excluded.

Our data collection for urine sample volumes and collection times were incomplete. The volume of urine sample obtained from the UCPs was $1-13 \mathrm{ml}$ (mean $4.9 \mathrm{ml}$ ) in 24 samples from each group. The time taken to complete a urine collection was recorded in 24/31 (77\%) in the replaced UCP group and in $23 / 37$ (62\%) in the single UCP group, and these proportions were not significantly different $(\mathrm{p}=0.18)$. There was a non-significant trend for a shorter collection time in the replaced UCP group (median 45 minutes, range 1240 minutes, $\mathrm{n}=24$ ) compared with the single UCP group (median 80 minutes, range 5-310 minutes, $\mathrm{n}=23$, $\mathrm{p}=0.056$ ) (two tailed Mann-Whitney U test).

To explore whether differences in sex distribution between the two collection methods (there was a relative excess of boys randomised to the single UCP group) could explain the very low rate of heavy mixed growth in the replaced UCP group $(3 \%)$, we compared the proportion of samples with heavy mixed growth between boys and girls within each group. We found no significant differences. In the replaced UCP group, the number of pads used for each child ranged between 1 and 7 (median 2). In $11 / 24$ (46\%), urine was successfully collected within 30 minutes (one pad) and a further $7 / 24$ (29\%) within one hour (two pads).

There were no adverse effects of the use of the enuresis alarm and no false alarms.

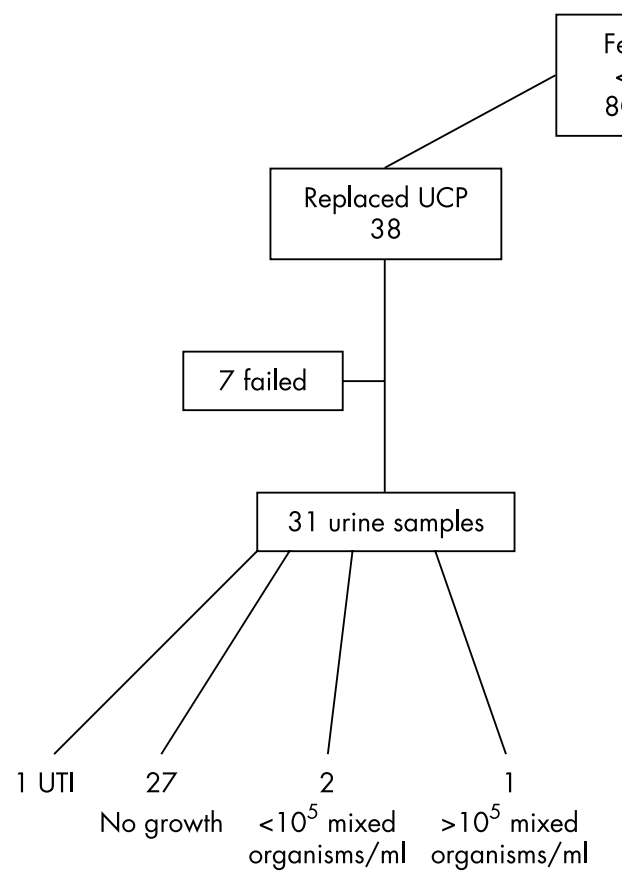

Figure 1 Flow chart showing the numbers of children through each stage of the randomised trial. UCP, Urine collection pad; UTI, urinary tract infection. 


\section{DISCUSSION}

In this study we have shown that, by replacing the UCP with a new pad every 30 minutes, the heavy $\left(>10^{5} / \mathrm{ml}\right)$ mixed growth contamination rate was significantly reduced from $29 \%$ to $3 \%$. This represents a simple and clinically useful improvement to the UCP method by substantially reducing the proportion of results from which UTI cannot be definitively excluded in this important age category. This finding supports our hypothesis that skin and faecal bacterial contamination of UCP samples is probably related to the contact time between the dry UCP and the perineal skin.

Compared with our previous study, this modified UCP method was superior to the clean catch method in which $12 \%$ of samples were heavily contaminated with mixed flora. ${ }^{2}$

We arbitrarily selected the time interval for replacing the UCP at every 30 minutes. It is possible that less frequent changing may yield similar results, but this has yet to be tested. In our previous study, ${ }^{2}$ in which heavy mixed growth contamination occurred in $27 \%$ of samples (very similar to the $29 \%$ in the single UCP method in this study), $74 \%$ of samples were obtained within 90 minutes. It seems likely therefore that the optimum interval between pad changes would be considerably less than 90 minutes.

Although the volume of urine retrieved from the UCPs was not recorded in all cases $(46 / 68,71 \%)$, recorded urine volumes were similar in both groups (about $5 \mathrm{ml}$ ) and were more than sufficient for bacteriological testing.

We continued to use the moisture sensitive alarm in combination with the UCP in both study groups as it considerably simplifies the method, even though our previous study failed to show any reduction in the proportion of samples heavily contaminated. There are two main practical benefits from using the audio alarm with UCPs. Firstly, the audio alarm immediately signals the presence of urine in the pad so the alarm method would be expected to slightly reduce the time required to collect the sample. Secondly, the child does not have to be disturbed in between each 30 minute UCP replacement to check for pad wetness. There were no false alarms-for example, from perspiration-and no adverse effects. The alarms are relatively inexpensive (about $£ 40$ ); the sensors are reusable.

There were two sources of potential bias in our study which require consideration. Firstly, our randomisation allocated a disproportionate number of boys to the single UCP group, and conversely a relative excess of girls to the replacement UCP group. We do not think this imbalance is sufficient to explain the lower rate of heavy mixed growth contamination of urine in the replacement UCP group for two reasons. Firstly, if the perineal skin in boys is more likely to contaminate the UCP urine, we would expect to find a significantly higher proportion of heavy mixed growth contamination results in boys compared with girls within the single UCP group; and no such difference was present. Secondly, on anatomical grounds, it might be expected that the female urethral meatus may be more accessible to perineal flora than the male urethra.

A second source of potential bias was that our data collection for the time taken to obtain the urine samples was incomplete in both groups. It is possible that there could have been a shorter time period between pad placement and voiding in the replaced UCP group and that it was this factor that reduced the amount of sample contamination. We cannot tell whether the trend towards quicker urine collection in the replaced UCP group that we observed was an artefact arising from incomplete data collection, or whether there is a mechanism operating whereby frequent opening of the nappy to replace the UCP stimulates bladder emptying. We think this is unlikely because in three quarters of children, urine was collected inside one hour-that is, only one UCP replacement.

We did not achieve complete data collection for recording the volume of urine obtained but the urine volumes obtained were more than adequate for laboratory bacterial culture (minimum $0.5 \mathrm{ml}$ ).

One limitation of the UCP method relevant to suspected UTI is that urine leucocyte counts by microscopy are significantly lower after pad collection because cellular material is partially retained in the UCP. ${ }^{2}$ However, this problem can be overcome with bedside, semiquantitative dipstick urinalysis to detect the presence of soluble leucocyte esterase.

Some common biochemical analyses have been validated from urine obtained from older style disposable nappies. ${ }^{8}$ However, further work is required to validate UCP samples for laboratory analyses other than bacterial culture before their use can be reliably extended to other clinical situations.

In this study we have shown that our modified UCP/alarm technique is a simple and clinically useful improvement to a child friendly urine collection method, which is reliable for diagnosing and excluding UTI in young children in hospital who are still in nappies. It has potential for use in outpatients clinics and primary care or at home and should be evaluated in these settings.

\section{ACKNOWLEDGEMENTS}

We thank Amanda Wilson who provided secretarial support to the study, our children's nursing staff who collected the urine samples, and Ferraris Medical Ltd UK who provided the enuresis alarms.

\section{Authors' affiliations}

S Rao, J Bhatt, C Houghton, P Macfarlane, Department of Child Health, Rotherham General Hospital, Rotherham, Yorkshire, UK

\section{REFERENCES}

1 Royal College of Physicians Research Unit Working Group. Guidelines for the management of acute urinary tract infection in childhood. J R Coll Physicians Lond 1991;25:36-42.

2 Macfarlane PI, Houghton C, Hughes C. Pad urine collection for early childhood urinary tract infection. Lancet 1999;354:571.

3 Vernon S, Redfearn A, Pedler SJ, et al. Urine collection on sanitary towels. Lancet 1994;334:612.

4 Lewis J. Clean catch versus urine collection pads: a prospective trial. Paediatr Nurs 1998; 10:15-16.

5 Feasey S. Are Newcastle urine collection pads suitable as a means of collecting specimens from infants? Paediatr Nurs 1999;2:17-21.

6 Liaw LCT, Nayar D, Pedler SJ, et al. Home collection of urine for culture from infants by three methods: survey of parents' preferences and bacterial contamination rates. BMJ 2000;320:1312-13.

7 Rao S, Houghton C, Macfarlane PI. A new urine collection method; pad and moisture sensitive alarm. Arch Dis Child 2002;86(suppl 1):A72(G220).

8 Ahmad T, Vickers D, Campbell S, et al. Urine collection from disposable nappies. Lancet 1991;338:674-6. 